\title{
0 Traumatic Anterior Ethmoidal Artery \\ Case \\ Report Pseudoaneurysm with Repeated Epistaxis Treated by Transarterial Embolization: A Case Report
}

Yuki Kuranari, ${ }^{1}$ Takenori Akiyama, ${ }^{1}$ Kaoru Yanagisawa, ${ }^{1}$ Dai Kamamoto, ${ }^{1}$ Katsuhiro Mizutani, ${ }^{1}$ Satoshi Takahashi, ${ }^{1}$

Shin Saito, ${ }^{2}$ Hiroyuki Ozawa, ${ }^{2}$ Kaoru Ogawa, ${ }^{2}$ and Kazunari Yoshida ${ }^{1}$

Objective: Pseudoaneurysms of the internal carotid artery (ICA) and sphenopalatine artery (SPA) are recognized as sources of arterial epistaxis following head and face trauma. However, epistaxis involving pseudoaneurysm of the anterior ethmoidal artery (AEA) is extremely rare.

Case Presentation: A 25-year-old man experienced massive epistaxis due to a ruptured traumatic pseudoaneurysm of the AEA. The patient had suffered head and face trauma in a car accident. CT showed fractures of the frontal, ethmoidal, and maxillary bones, and he was managed conservatively. Nine days after the injury, he had sudden, massive epistaxis. Angiography showed a right AEA aneurysm, which was treated successfully with transarterial embolization using n-butyl2-cyanoacrylate (NBCA).

Conclusion: Although pseudoaneurysm of the AEA is a rare cause of epistaxis, it is important to consider this diagnosis, in addition to pseudoaneurysm of the SPA and ICA, when a patient has massive arterial epistaxis following a traumatic skull base fracture, especially if the fracture is adjacent to the ethmoid sinus. Transarterial embolization using glue is a feasible therapeutic option for this condition.

Keywords > pseudoaneurysm, anterior ethmoidal artery, epistaxis, transarterial embolization

\section{Introduction}

Epistaxis due to rupture of traumatic pseudoaneurysms ${ }^{1)}$ with involvement of the ophthalmic artery (OphA) or its tributaries is rare, with only two reported cases in the literature. ${ }^{2,3)}$ Here, we describe a case of ruptured anterior ethmoidal artery (AEA) pseudoaneurysm with massive arterial

\footnotetext{
${ }^{1}$ Department of Neurosurgery, Keio University Hospital, Tokyo, Japan

${ }^{2}$ Department of Otorhinolaryngology, Keio University Hospital, Tokyo, Japan
}

Received: May 17, 2018; Accepted: August 7, 2018

Corresponding author: Yuki Kuranari. Department of Neurosurgery, Keio University Hospital, 35 Shinanomachi, Shinjuku-ku, Tokyo 160-8582, Japan

Email: yuki_kuranari@keio.jp

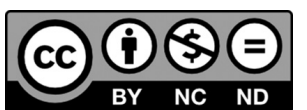

This work is licensed under a Creative Commons Attribution-NonCommercialNoDerivatives International License.

(C)2019 The Japanese Society for Neuroendovascular Therapy epistaxis. The aneurysm was treated successfully by transarterial embolization using n-butyl-2-cyanoacrylate (NBCA). Since epistaxis due to a ruptured pseudoaneurysm can be life-threatening, the clinical course and discussion of this case are useful for elucidating the etiology of this rare but important clinical entity.

\section{Case Presentation}

A 25-year-old man experienced head and face trauma following a car accident. On admission to a nearby hospital, he was conscious with no neurologic deficits, but had periorbital contusions. Head CT immediately after the injury revealed multiple bone fractures, including frontal, ethmoidal, and maxillary bones. After 9 days of conservative observation, the patient had a sudden and massive epistaxis of more than $500 \mathrm{~mL}$. His hemoglobin dropped to $7.4 \mathrm{~g} / \mathrm{dL}$. CTA and DSA demonstrated a $4 \mathrm{~mm}$ aneurysm of the right AEA (Fig. 1). Epistaxis recurred and the aneurysm size increased to $6 \mathrm{~mm}$ (Fig. 2). Epistaxis temporarily decreased 

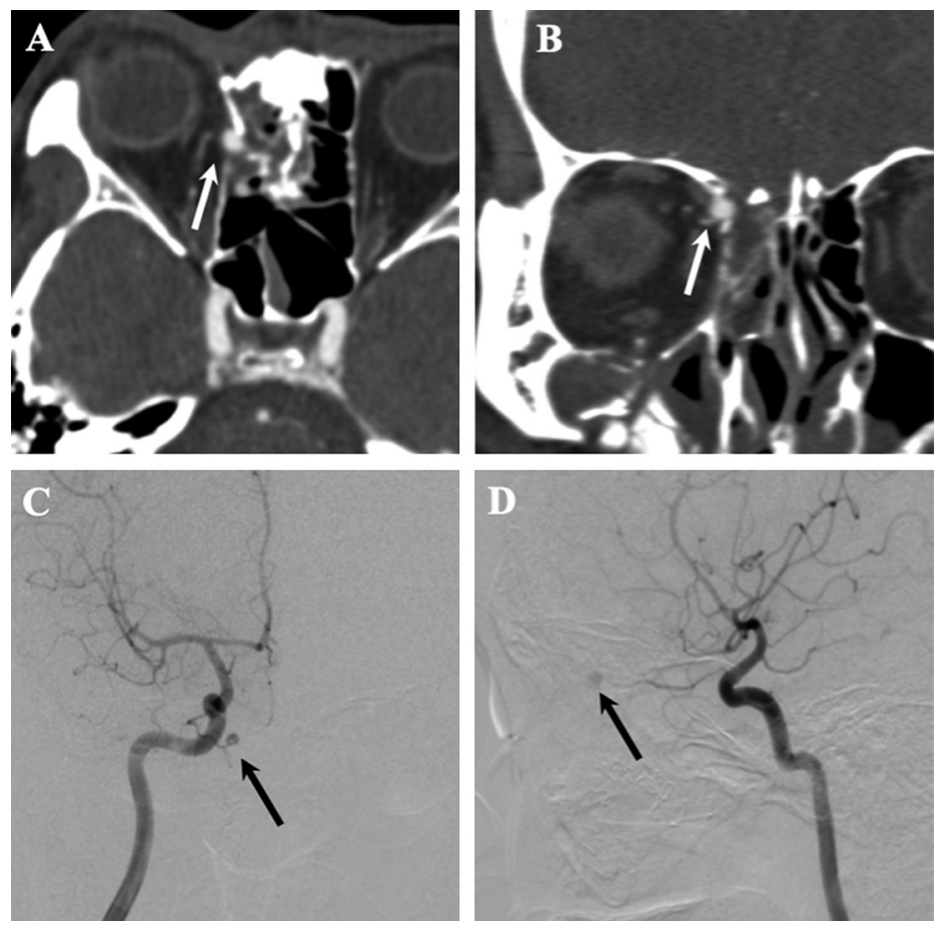

Fig. 1 Enhanced CT showing the aneurysm at the fracture site (arrow) of the outer wall of the ethmoidal sinus; (A) axial view, (B) coronal view. DSA of the right ICA showing the aneurysm (arrow) with a diameter of $4 \mathrm{~mm}$ at the peripheral site of the OphA; (C) anteroposterior view, (D) lateral view. ICA: internal carotid artery; OphA: ophthalmic artery

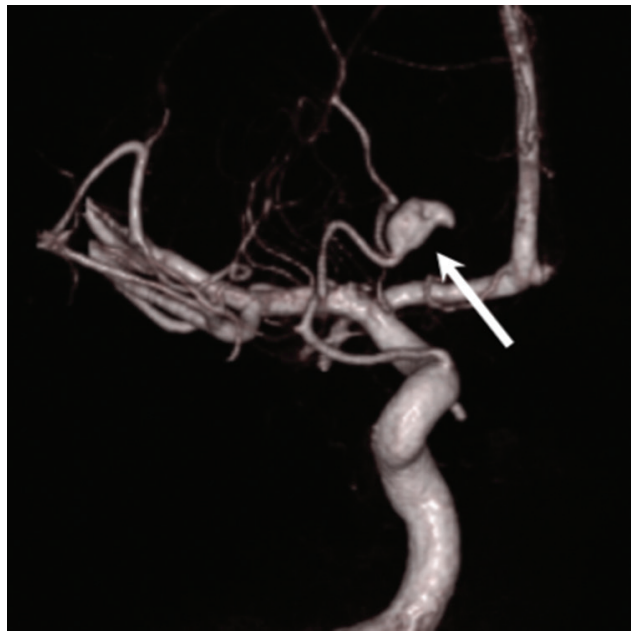

Fig. 2 Reconstructed enhanced CT image showing aneurysm enlargement (arrow).

after nasal packing, but easily restarted when the packing materials slipped. The patient was transferred to our institution for further treatment.

Angiography was performed (Fig. 3A), and the AEA showed anastomosis with the facial artery distal to the aneurysm (Fig. 3B). The location of the aneurysm was consistent with the bone fracture where the AEA typically penetrates the bone and reaches the ethmoidal sinus. The aneurysm lacked an aneurysmal neck and its shape was consistent with a pseudoaneurysm (Figs. 2 and 3A).

Transarterial embolization using NBCA was performed. Under general anesthesia, a 6 Fr guiding sheath (Asahi Intecc Co., Ltd, Aichi, Japan) was inserted via the right femoral artery into the right internal carotid artery (ICA). A Cerulean DD6 (Medikit co. 1td, Tokyo, Japan) was advanced through the guiding catheter into the cavernous portion of the artery. Since we initially considered embolization with both coils and glue, an Excelsior SL-10 microcatheter (Stryker, Kalamazoo, MI, USA) was used to approach the lesion. The microcatheter was delivered into the right AEA through the OphA in a wedged position to maintain a safe margin from the central retinal artery in case of reflux. We embolized the aneurysm and the AEA proximal and distal to the aneurysm using 25\% NBCA (0.2 mL, room temperature) (Fig. 3C and 3D). After treatment, the aneurysm disappeared completely (Fig. 3E), and the patient was discharged 8 days later. Post-interventional radiology and an ophthalmological examination revealed no vision loss or eye movement disorders. There was no recurrence of the aneurysm at 60-day follow-up CTA. 

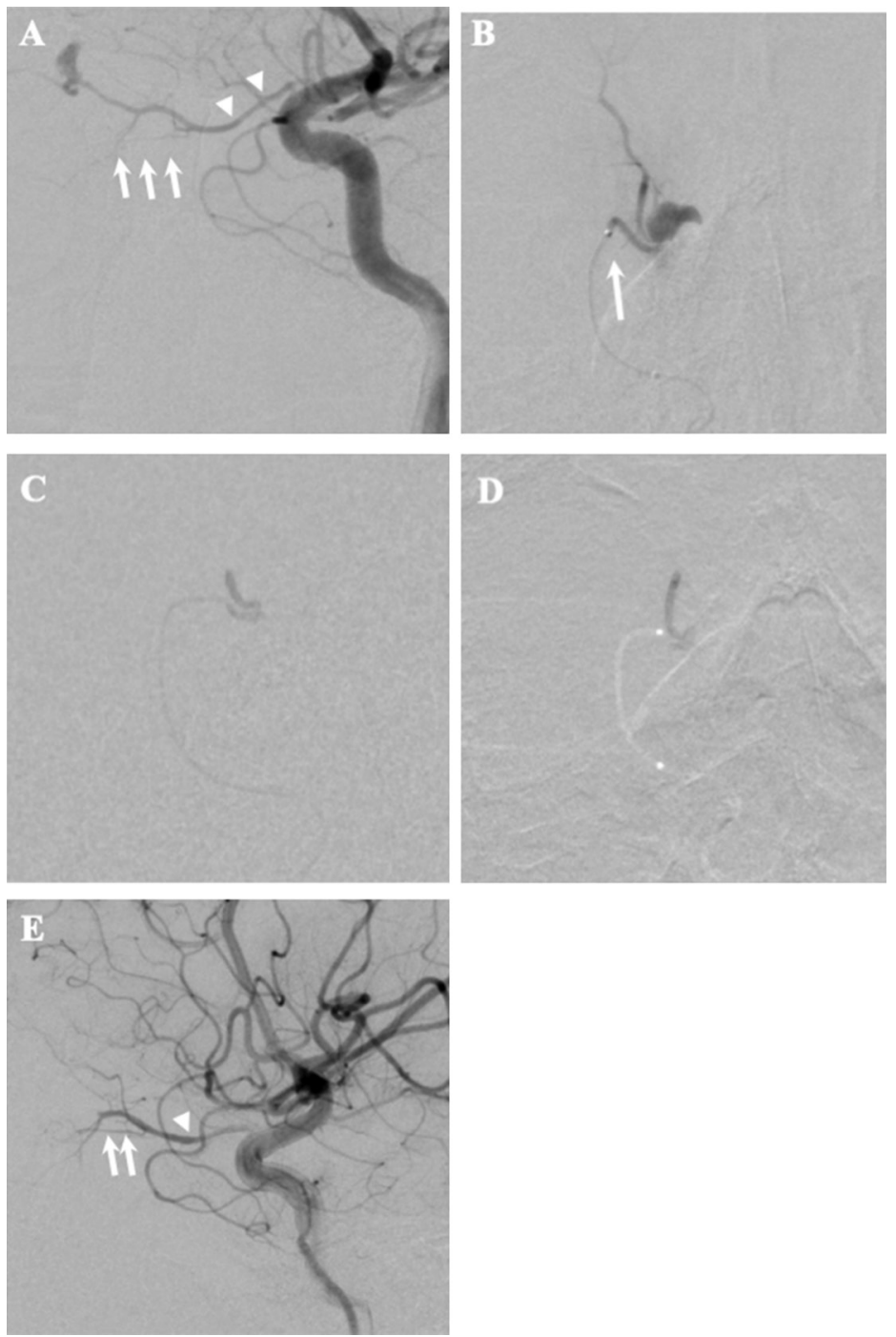

Fig. 3 Intraoperative DSA. (A) Lateral view angiography showing the OphA (arrowheads) and central retinal artery (arrows). (B) Anteroposterior view $\left(\mathrm{CAU} 26^{\circ}\right.$ ) anastomosis of the AEA with the facial artery (arrow). (C and D) Anteroposterior view (CAU $26^{\circ}$ ) images during NBCA injection to the distal to the proximal site of the aneurysm. (E) Lateral view postembolization angiography showing aneurysm disappearance and blood flow to the OphA (arrowhead) and central retinal artery (arrows). AEA: anterior ethmoidal artery; CAU: caudal angulation; NBCA: N-butyl-2-cyanoacrylate; OphA: ophthalmic artery

\section{Discussion}

Epistaxis after rupture of a pseudoaneurysm may be intractable and life-threatening. Early identification and hemostasis are thus essential. The majority of reported traumatic pseudoaneurysms causing arterial epistaxis involve the ICA and sphenopalatine artery (SPA). ${ }^{1)}$ However, there are reports of rare origins, such as the basilar and middle meningeal arteries. ${ }^{4,5)} \mathrm{A}$ review of the literature revealed only six reported cases of traumatic pseudoaneurysm of the OphA and its tributaries (Table 1), ${ }^{2,3,6-9)}$ only two of which had epistaxis. ${ }^{2,3)}$ In one case, a gunshot wound had injured the cranial base and the OphA. ${ }^{2}$ The other case involved long-term nasogastric intubation, which had injured the distal portion of the AEA running through the nasal cavity. ${ }^{3)}$

In our patient, fracture of the lateral wall of the ethmoid sinus adjacent to the anterior ethmoid foramen, which the AEA traversed with the direct blunt injury to the AEA, resulted in the formation of pseudoaneurysm. However, pseudoaneurysm formation in this location following blunt craniofacial trauma has not been reported previously. This location is susceptible to pseudoaneurysm formation 


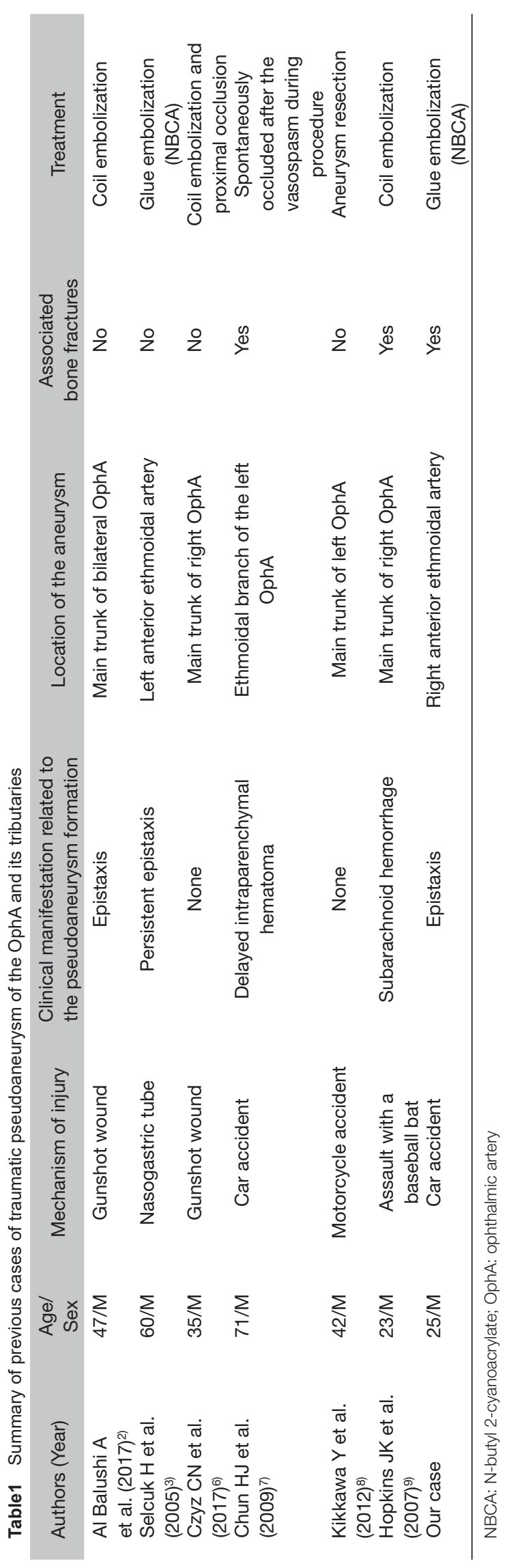

following trauma because the AEA penetrates and is fixed to the bone. In cases of anterior skull base fracture and massive epistaxis, especially when the fracture is adjacent to the ethmoid sinus, the AEA, ICA, and branches of the external carotid artery should be examined carefully.

Our patient was treated successfully with transarterial embolization using NBCA. The definitive treatment of pseudoaneurysm is occlusion of the parent vessel. ${ }^{10)}$ Proximal occlusion can reduce flow into the pseudoaneurysm, thus reducing the risk of re-rupture. However, the AEA is not an end artery and may anastomose with the facial artery, similar to the current case. It should be noted that the possibility of rupture remains after proximal occlusion. In our patient, the aneurysm was in a relatively distal portion of the tortuous AEA; therefore, we also considered the use of a flow guide catheter in addition to an Excelsior SL-10 microcatheter. We initially used an Excelsior SL-10 microcatheter preparing the coil embolization with various types of the coil. We intended to change it to a flow guide catheter when it was difficult to approach the lesion. Occlusion using glue is another alternative, ${ }^{1)}$ but is generally contraindicated for lesions in the OphA or its branches due to the risk of glue migration into the central retinal artery, which may result in permanent blindness. ${ }^{11)}$ While previous studies have reported proximal embolization using platinum coils instead of glue injection into the OphA, 2,4,6,10) advanced endovascular techniques allow transarterial embolization involving the branches of the OphA, especially in cases with anterior cranial fossa dural arteriovenous fistulas. ${ }^{12,13)}$ During glue injection via the tributaries of the OphA, it is essential to ensure a safe margin between the injection point and the central retinal artery, and dangerous anastomosis with the ICA via the deep recurrent OphA. Advanced training is required to perform the injection, which involves continuous glue injection to achieve complete occlusion of the aneurysm and the proximal parent artery and to prevent reflux to dangerous arteries. Although transarterial embolization using glue is feasible for the treatment of a pseudoaneurysm of the OphA or its tributaries, the evaluation of the arterial anatomy, safety margins, and anastomoses with other intracranial or extracranial arteries as well as mastery of the embolization technique are needed for this treatment to be safe and effective.

\section{Conclusion}

Although traumatic pseudoaneurysm of the AEA is very rare, it can cause severe epistaxis. In patients with craniofacial 
trauma with skull base fracture and massive epistaxis, the existence of a pseudoaneurysm in the AEA, in addition to the more common sites, should be considered. Transarterial embolization with NBCA is an effective treatment for this condition.

\section{Disclosure Statement}

There are no conflicts of interest to disclose regarding this paper.

\section{References}

1) Zhang Cw, $\mathrm{Xie} \mathrm{Xd}$, You $\mathrm{C}$, et al: Endovascular treatment of traumatic pseudoaneurysm presenting as intractable epistaxis. Korean J Radiol 2010; 11: 603-611.

2) Al Balushi A, Kitchener J, Edgell RC: Bilateral ophthalmic artery dissecting aneurysms presenting with recurrent epistaxis. J Vasc Interv Neurol 2017; 9: 14-16.

3) Selcuk H, Soylu N, Albayram S, et al: Endovascular treatment of persistent epistaxis due to pseudoaneurysm formation of the ophthalmic artery secondary to nasogastric tube. Cardiovasc Intervent Radiol 2005; 28: 242-245.

4) Li T, Zhang Y, Gu Z, et al: Traumatic pseudoaneurysm of the basilar artery presenting with fatal epistaxis: a rare case report. Brain Inj 2013; 27: 1316-1319.

5) Gu J, Lu J, Wang X, et al: Traumatic middle meningeal artery pseudoaneurysms presenting with intractable epistaxis: a rare case report and review of literature. Case Reports in Clinical Medicine 2015; 4: 28-31.
6) Czyz CN, Piehota PG, Strand AT, et al: Post-traumatic ophthalmic artery pseudoaneurysm from orbital projectile. Can J Ophthalmol 2017; 52: e130-e132.

7) Chun HJ, Yi HJ: Traumatic extracranial pseudoaneurysm on the peripheral ophthalmic artery presenting as delayed intraparenchymal hematoma: case report. Surg Neurol 2009; 71: 701-704.

8) Kikkawa Y, Natori Y, Sasaki T: Delayed post-traumatic pseudoaneurysmal formation of the intracranial ophthalmic artery after closed head injury. Case report. Neurol Med Chir (Tokyo) 2012; 52: 41-43.

9) Hopkins JK, Shaibani A, Ali S, et al: Coil embolization of posttraumatic pseudoaneurysm of the ophthalmic artery causing subarachnoid hemorrhage. Case report. J Neurosurg 2007; 107: 1043-1046.

10) Pelliccia P, Bartolomeo $M$, Iannetti $G$, et al: Traumatic intra-sphenoidal pseudoaneurysm lodged inside the fractured sphenoidal sinus. Acta Otorhinolaryngol Ital 2016; 36: 149-152.

11) Kakarla UK, Deshmukh VR, Zabramski JM, et al: Surgical treatment of high-risk intracranial dural arteriovenous fistulae: clinical outcomes and avoidance of complications. Neurosurgery 2007; 61: 447-457; discussion 457-459.

12) Agid R, Terbrugge K, Rodesch G, et al: Management strategies for anterior cranial fossa (ethmoidal) dural arteriovenous fistulas with an emphasis on endovascular treatment. J Neurosurg 2009; 110: 79-84.

13) White DV, Sincoff EH, Abdulrauf SI. Anterior ethmoidal artery: microsurgical anatomy and technical considerations. Neurosurgery 2005; 56: 406-410. 